\title{
La méthode Berlitz : entre méthode naturelle et méthode directe
}

The Berlitz method: between the natural method and the direct method

Javier Suso López

\section{(2) OpenEdition}

Édition électronique

URL : https://journals.openedition.org/dhfles/7868

DOI : $10.4000 /$ dhfles.7868

ISSN : 2221-4038

Éditeur

Société Internationale pour l'Histoire du Français Langue Étrangère ou Seconde

Édition imprimée

Date de publication : 1 décembre 2020

Pagination : 183-202

ISSN : 0992-7654

Référence électronique

Javier Suso López, «La méthode Berlitz : entre méthode naturelle et méthode directe », Documents pour l'histoire du français langue étrangère ou seconde [En ligne], 64-65 | 2020, mis en ligne le 24 février 2021, consulté le 24 avril 2023. URL : http://journals.openedition.org/dhfles/7868 ; DOI : https:// doi.org/10.4000/dhfles.7868

Ce document a été généré automatiquement le 24 avril 2023

Tous droits réservés 


\title{
La méthode Berlitz : entre méthode naturelle et méthode directe
}

\author{
The Berlitz method: between the natural method and the direct method
}

\author{
Javier Suso López
}

\section{Introduction}

Rappelons tout d'abord l'anecdote qui raconte la naissance de la méthode Berlitz :

\begin{abstract}
Maximilian Delphinius Berlitz (Mühringen, Württemberg, 1852 - New York, 1921). Après un séjour en France, il s'installe aux États-Unis en 1870 où il enseigne comme professeur de français et d'allemand, avant de prendre en 1878 la direction du Warner Polytechnic College. Ne pouvant plus faire face à toutes ses obligations, il engage un Français, Nicolas Joly, afin de le seconder dans ses cours de français avant de se rendre compte que celui-ci ne parle pas un mot d'anglais. Néanmoins, bien qu'il dispense ses cours entièrement en français, sans traduction, les résultats sur les élèves s'avèrent extrêmement probants, Joly utilisant des gestes, des attitudes et des objets pour faciliter la compréhension et susciter le dialogue [...] ${ }^{1}$. Berlitz used this experience to develop the Berlitz Method, for which only the language to be taught is spoken from the first day of class. Students rely on the same techniques Joly used, rather than translation, to gather meaning and learn grammar and vocabulary ${ }^{2}$.
\end{abstract}

2 L'idée qui ressort de ces présentations est que Berlitz, s'inspirant de la façon de faire de Nicolas Joly, inventa sa méthode de toutes pièces dès 1878 et la mit telle quelle en oeuvre, remportant un énorme succès et créant un vaste réseau d'écoles Berlitz ${ }^{3}$. Cette image installe le mythe du "génie » ou de l'« inventeur »; nous allons montrer quelles influences et quelle évolution subit la méthode Berlitz au cours des 35 premières années (1882-1915) de son existence. 


\section{Matériels langagiers de la méthode Berlitz et corpus}

3 Nous avons fixé le terminus a quo en 1882a car c'est à cette date qu'est publiée la première édition de la Méthode pour l'enseignement de la langue française dans les écoles Berlitz (MB1-1882) ; quant au terminus ad quem (1915), nous considérons qu'à cette date la méthode Berlitz est déjà établie de manière substantielle et solide. Notre étude porte uniquement sur la méthode Berlitz pour l'enseignement de la langue française: les manuels Berlitz comprennent les principales langues européennes (français, anglais, allemand, espagnol, italien...), qui déclinent la même «méthode » quelle que soit la langue enseignée; cependant, c'est pour la langue française qu'existe une plus grande variété de manuels et d'outils complémentaires.

4 Pour mieux comprendre le choix de notre corpus, il faut indiquer que les ouvrages de la méthode Berlitz pour le français comprennent non seulement des manuels (divisés en niveaux 1 et 2, destinés aux enfants et aux adultes, pour le public « américain » ou bien pour d'autres nations, pour des contextes d'auto-apprentissage...) dont les éditions sont revues, corrigées et/ou augmentées au fil des ans, jusqu'à nos jours, mais également une panoplie d'outils complémentaires, tels que :un traité de la prononciation, une grammaire pratique en 4 volumes, une collection de nouvelles et de comédies, un ouvrage sur la littérature française, une clé (key), un traité sur le genre des substantifs, des observations pratiques pour les étrangers...

5 Pour le corpus, nous n'avons gardé que les manuels pour l'enseignement du français adressés à un public d'apprenants adolescents et adultes. Les manuels Berlitz que nous avons consultés et qui constituent notre corpus sont ainsi les suivants, classés par ordre chronologique :

6 -Berlitz, M.D. \& Dubois, E. (1882a). Méthode pour l'enseignement de la langue française dans les écoles Berlitz. Première partie. 45 pages. Boston, Mass. [MB1-1882a] ${ }^{4}$.

7 -Berlitz, M.D. \& Dubois, E. (1882b). Premier livre de lecture des écoles Berlitz. 120 pages. Boston, Mass. : Imp. Schriftgriesser. [PremierLivre-1882b]

8 -Berlitz, M.D. (1897). Méthode Berlitz pour l'enseignement des langues modernes. Nouvelle édition, revue et augmentée. Partie française. Premier livre. 85 pages $(82+$ Tableau synoptique des verbes). Préface en anglais. Éditions consultées : 1897 et 1903. Madison Square, New York: Berlitz et $C^{\text {ie }} ; 17$ Blumenthal St., Berlin: Siegfried Cronbach ; 27 Avenue de l'Opéra, Paris et 231 Oxford Street, London: Berlitz School ${ }^{5}$. [MB1-[1887] 1897/1903].

9 -Berlitz, M.D. ( $\left.{ }^{16} 1901\right)$. Méthode Berlitz pour l'enseignement des langues modernes. Nouvelle édition, revue et augmentée. Partie française. Deuxième livre. 154 pages. Berlin: Siegfried Cronbach; New York: Berlitz \& Co.; Paris: The Berlitz School ; Londres: The Berlitz School; Copenhague: Wilhelm Tryde; St. Pétersbourg \& Moscou: M.O. Wolff. [MB2- $\left.{ }^{16} 1901\right]$.

10 -Berlitz, M. D. ([1902] 1924). Deuxième livre pour l'enseignement des langues modernes: Partie française pour adultes. 154 pages. Édition consultée : 1924, revue et complètement remaniée. [MBadultes2-[1902]1924].

11 -Berlitz, M.D. ([1903] 1905). Premier livre pour l'enseignement des langues modernes : partie française pour adultes. New York: M.D. Berlitz; Paris : The Berlitz School ; London : The Berlitz School; Berlin : Siegfried Cronbach,. 102 pages + Appendice : Tableau synoptique 
des formes verbales. Éditions consultées : 1905 (nouvelle édition américaine, revue et complètement remaniée) et $1907^{6}$. [MBadultes1-[1903]1905].

-Berlitz, M.D. (1915). Méthode Berlitz pour l'enseignement des langues modernes. Nouvelle édition, revue et augmentée. Berlin : Siegfried Cronbach. 122 pages (dont l'Appendice 1) + Appendice II: Tableau synoptique des formes verbales. Édition consultée: [MB1$\left.[1915]^{285} 1924\right]$.

\section{Sources, évolution et caractéristiques de la méthode Berlitz}

13 Selon les présentations actuelles insérées dans les pages web des écoles Berlitz (voir citations ci-dessus), la méthode Berlitz s'inscrit dans le courant de la «méthode directe » (désormais $\mathrm{MD})^{7}$, partageant les mêmes procédés méthodologiques de classe : elle installe les élèves dans la langue cible et rejette l'usage de la langue maternelle (L1) et donc la traduction, s'appuyant sur des gestes et des objets pour faciliter la compréhension et susciter le dialogue.

14 Cependant, dans la préface à MB1-[1887] 1897/1903, Berlitz - au-delà de l'anecdote reprise au début de cet article sur l'origine de sa méthode - utilise comme référence de son "système d'instruction langagière » la méthode naturelle mise en œuvre par le professeur Gottlieb Heness :

The Berlitz Method is based on a System of language instruction generally called the 'Natural Method' (first used by Professor Henness). In it the pupil is acquainted with the foreign tongue, not by translation, which is abandoned altogether, but by conversation al exercises in the new language (MB1-[1887]1897/1903).

\section{La méthode naturelle aux USA et son influence sur la méthode Berlitz}

15 Ce n'est pas notre objet de traiter la question de l'arrivée du mouvement naturel d'enseignement des langues et de son développement aux USA, mais une brève contextualisation historique nous semble nécessaire. Irene Finotti (2010) et Anthony P.R. Howatt \& Richard Smith (2014), s'occupent de cette question de manière suffisante par rapport aux objectifs de notre étude. Non seulement Gottlieb Heness, de nationalité allemande et parlant également français mais aussi Lambert Sauveur avaient émigré aux États-Unis ${ }^{9}$ où ils s'étaient établis dans les années 1860-70 et avaient créé des académies de langues, publié des manuels... D'autre part, «nel 1880 il francese Gouin pubblicò L'art d'enseigner et d'étudier les langues che all'epoca fu totalmente ignorata in Francia [...], ma riscosse un notevole successo in Germania, ed investì poi l'Inghilterra e l'America » (Titone 1985 : 69). Dans sa monographie, Finotti «traite spécifiquement des propositions de Sauveur et des rapports qu'elles peuvent entretenir avec celles de Berlitz » (Besse 2010 : 234) ; elle met en relief le rôle de Heness et de Sauveur (qui doit beaucoup à Heness, selon Finotti) dans la propagation d'une nouvelle méthode d'enseignement des langues aux USA (Finotti $2010: 12)^{10}$.

Grâce à ses cours et à ses ouvrages, "Sauveur jouit dans le pays qui l'accueille d'une certaine renommée, voire d'une popularité immense' d'après Geddes (1930: 252), auteur ayant fréquenté les cours de Sauveur dans sa jeunesse, et qui le considère comme 'le principal interprète' [...] de la méthode naturelle » (Finotti $2010: 11)$. Howatt 
\& Smith reconnaissent également le rôle de Heness et de Sauveur dans l'introduction de la méthode naturelle aux États-Unis (Howatt \& Smith 2014 : 83-84) ${ }^{11}$.

La démarche méthodologique de Berlitz doit donc beaucoup à ces professeurs de langues (Berlitz ne reconnaît, en passant, que l'influence de Heness, comme nous l'avons vu) et à la méthode naturelle qu'ils mettaient en œuvre dans leurs cours et leurs manuels; en tout cas si la méthode naturelle fut « first used by Professor Heness » aux USA, comme le constate Erwin Escher dans les conclusions de son étude (1920: 298-299), il faut ajouter que Heness ne faisait que divulguer les idées d'une approche naturelle de l'enseignement des langues vivantes, défendues en Allemagne dans les années 1860 par Karl Griep et A. F. Louvier et mises en pratique, avec des manuels d'enseignement (voir Escher 1920: 298 et Reinfried 1990: 126-156); en France, la méthode naturelle avait été recommandée par le ministre de l'instruction publique, Victor Duruy, en 1863...

Ceci dit, il nous semble important ainsi de distinguer, avec Howatt \& Smith, le courant de la méthode naturelle qui, "in private language schools in the United States, involved the development of a methodology that made foreign languages accessible to the adult population generally - not only a classically educated elite - and focused on the teaching of conversation» et le Mouvement de la Réforme ("the pan-European Reform Movement ", pour reprendre l'appellation utilisée par ces auteurs), qui se développe en Europe à partir des années 1882.

\section{Évolution de la méthode Berlitz}

La méthode Berlitz se transforme au fil des années : nous allons exposer dans les pages qui suivent l'évolution que connaît sa méthode à travers l'analyse des manuels et des prises de position des Préfaces.

\section{Les manuels Berlitz}

20 Nous allons commencer notre analyse par le premier livre de la Méthode pour l'enseignement de la langue française, en mettant en parallèle les éditions suivantes: MB1-1882a, PremierLivre-1882b, MB1-[1887]1897/1903, [MBadultes1-[1903]1905] et MB1$[1915]^{285} 1924$.

21 Les tableaux suivants permettent de mieux voir l'évolution produite quant à la texture et l'organisation des manuels :

\begin{tabular}{|l|l|l|l|}
\hline Titre & Préface & Nombre de leçons & Nombre de pages \\
\hline MB1-1882a & $\begin{array}{l}\text { en anglais : } \\
\text { 1 page }\end{array}$ & 20 leçons & 45 \\
\hline \multirow{2}{*}{ PremierLivre-1882b } & $\begin{array}{l}\text { En français : une } \\
\text { demi-page }\end{array}$ & $\begin{array}{l}\text { Lectures accompagnées d'exercices } \\
\text { de compréhension }\end{array}$ & 120 \\
\hline \multirow{2}{*}{$\begin{array}{l}\text { MB1-[1887] } \\
1897 / 1903\end{array}$} & en anglais : & Leçons préparatoires : 14 leçons & $35(9-44)$ \\
\cline { 2 - 4 } & 5 pages & Elementary reading and conversations & $32(51-82)$ \\
\cline { 2 - 4 }
\end{tabular}




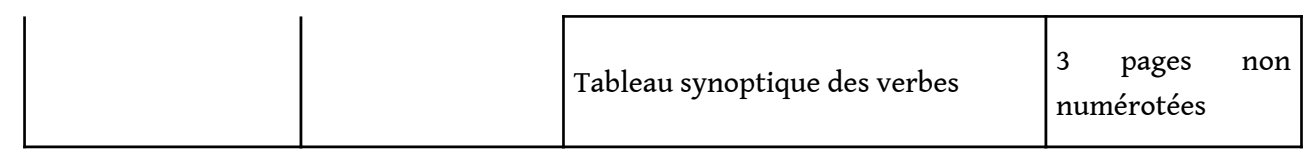

Tableau 1

\begin{tabular}{|c|c|c|c|}
\hline \multirow{3}{*}{$\begin{array}{l}\text { MBadultes1- } \\
{[1903] 1905}\end{array}$} & \multirow{3}{*}{$\begin{array}{l}\text { en } \\
\text { français : } \\
5 \text { pages }\end{array}$} & $\begin{array}{l}\text { Partie préparatoire (leçons de choses) : } 14 \\
\text { leçons }\end{array}$ & $38(11-49)$ \\
\hline & & Morceaux de lectures et de conversation $\left(^{*}\right)$ & $51(51-102)$ \\
\hline & & $\begin{array}{l}\text { Appendice: Tableau synoptique des } \\
\text { verbes }\end{array}$ & $\begin{array}{l}3 \text { pages non } \\
\text { numérotées }\end{array}$ \\
\hline
\end{tabular}

TABLEAU 2. ${ }^{\star}$ ) Cet intitulé du tableau synoptique devient Morceaux élémentaires dans le corps du texte

\begin{tabular}{|c|c|c|c|}
\hline \multirow{4}{*}{$\begin{array}{l}\text { MB1- } \\
{[1915]} \\
{ }^{285} 1924\end{array}$} & \multirow{4}{*}{$\begin{array}{l}\text { en } \\
\text { français : } \\
6 \text { pages }\end{array}$} & Partie préparatoire (leçons de choses) : 12 leçons & $29(13-44)$ \\
\hline & & Morceaux de lecture et de conversation & $57(39-116)$ \\
\hline & & $\begin{array}{l}\text { Appendice I: Exemples et exercices } \\
\text { complémentaires }\end{array}$ & $17(119-136)$ \\
\hline & & Appendice II : Tableau synoptique des verbes & $\begin{array}{lr}3 & \text { pages non } \\
\text { numérotées } & \end{array}$ \\
\hline
\end{tabular}

Tableau 3

22 Ces modifications extérieures consistent principalement en ceci :

23 - la MB1-1882a et le Premier Livre de lectures (1882b) sont signés par deux auteurs : M.D. Berlitz et E. Dubois ${ }^{12}$.

24 - la préface prend de l'ampleur : d'abord rédigée en anglais (éditions de1882 et de 1887), elle est rédigée en français dès que les écoles Berlitz s'installent en Europe (voir note 3).

25 - le nombre total de pages augmente au fur et à mesure : 45, 82, 102 et 116 pages.

26 - le nombre des leçons « préparatoires » diminue : 20 leçons en 1882a, 14 en 1897 et en 1903, 12 en 1915. Elles occupent également moins de pages : 42, 35, 38 et 29.

27 - les lectures et les conversations qui constituent un livre à part (1882b, 120 pages), sont intégrées dans les manuels postérieurement, et font l'objet d'une forte sélection, ne gardant que les textes en langue courante : 32 pages en 1897, 51 en 1903 et 57 pages en 1915.

28 - des exemples et des exercices complémentaires sont introduits en 1915 (Appendice I, qui comprend 37 pages).

29 Le contenu de l'enseignement lui-même (l'« object teaching », ou " substance of each lesson », pour employer les termes de Berlitz) ne subit pas de grandes variations. La partie préparatoire (leçons de choses) reprend le même contenu le long des quatre éditions consultées, quant aux sujets permettant de regrouper le vocabulaire et quant aux points grammaticaux, même si l'ordre de présentation du contenu change dans 
chacune des quatre éditions analysées. Bien sûr, le nombre plus limité de leçons (vingt leçons en 1882 ; douze en 1915) entraîne une diminution du nombre de mots utilisés dans la partie préparatoire et les exercices correspondants. Il faudrait faire une analyse plus détaillée pour déterminer le nombre total de mots proposés. Quelle est la diminution effective opérée par Berlitz (1915:6) et quels termes du lexique sont concernés? Certains mots sont proposés de manière active - donc, réutilisés dans les conversations, dans une « possession effective » dont parle Joseph Firmery (1902:331), en reprenant l'expression utilisée par la Circulaire ministérielle du 15 novembre 1901 -, et d'autres de manière passive, pour leur compréhension uniquement, dans les lectures complémentaires?

Disons à cet égard que l'une des caractéristiques de la MD, face à la démarche basée sur les «leçons des choses ", est précisément la diminution des mots à apprendre, surtout dans la première phase de l'enseignement :

Pour parler - je veux dire par là, pour exprimer ses besoins et ses impressions les plus immédiates, pour exprimer ce qui est proprement du domaine de la conversation usuelle - pour parler, il n'est besoin que d'un vocabulaire extrêmement restreint (Firmery $1902: 332$ ).

\section{La progression dans l'acquisition/apprentissage de la langue étrangère}

31 C'est la mise en progression du contenu de l'apprentissage qui révèle mieux encore l'évolution de la méthode Berlitz. Nous devons distinguer ici la progression dans les contenus langagiers (c'est-à-dire, l'ordre de présentation de l'objet d'apprentissage : vocabulaire, grammaire) et la progression dans l'acquisition, donc, considérée du point de vue psycholinguistique.

32 La progression des contenus lexicaux est établie, dès MB1-1882a et PremierLivre-1882b, autour des « choses » qui s'offrent à la vue des élèves dans la salle de classe : les objets de la salle de classe, les gestes, les couleurs, la place et la position (localisation dans l'espace), les parties du corps, la quantité... et les actions qui peuvent être réalisées à partir de ces objets ${ }^{13}$. Une série de modifications se produisent au fur et à mesure des éditions, fruits de l'expérience de l'enseignement sans que, globalement, cet ordre ne soit remis en question. Il répond aux contraintes de la pédagogie basée sur les leçons des choses : c'est sur les objets environnants, présents à la vue des élèves que les leçons s'articulent; les manuels de Berlitz, sauf Méthode Berlitz pour l'enseignement des langues modernes. Édition illustrée pour les enfants (1897), ne contiennent pas d'images (ou des dessins) pour orienter le regard des élèves en dehors de la salle de classe, comme le feront certains méthodologues directs postérieurs à travers des tableaux. Ainsi, Alge ${ }^{5} 1896$, et Alge \& Rippmann 1904 incorporent dans leurs manuels les gravures des quatre saisons éditées par Eduard Hölzel à Vienne ; de même, les tableaux Delmas deviendront un outil complémentaire utilisé par des nombreux professeurs ${ }^{14}$.

Malgré l'absence d'images, la démarche de Berlitz n'est pas différente de celle d'autres méthodologues directs. Ernest Rochelle détaille d'une façon très concrète chacun des pas de ces premières phases de l'enseignement : interpellation des élèves, questions portant sur les objets environnants, étude du corps humain et des vêtements, recours au tableau mural... (Rochelle 1906: 9-10); c'est le cas également de Nathalie Wickerhauser :

La manière de remuer cette matière dans la psyché de l'élève doit être psychologique; c'est-à-dire, il faut arranger la matière par groupes, en faisant succéder un sujet après un autre d'après un certain système logique : nombres, 
temps, lieu, etc.; ou bien école, maison, rue, village, ville, etc. Ainsi on peut faire parler l'élève d'une manière spontanée, comme s'il parlait des mêmes sujets dans sa langue maternelle; cela veut dire : le maître doit développer la conversation selon les idées des élèves et non pas les forcer d'accepter les siennes (1907:223).

En outre, un ordre de présentation des contenus de grammaire, totalement nouveau également, accompagne cette nouvelle organisation du vocabulaire, et ceci, dès le premier manuel de Berlitz (1882a). Comme nous l'avons indiqué dans une autre étude (Suso \& Valdés 2020 : à paraître), la mise en rapport entre le vocabulaire et les règles de grammaire constitue une des réalisations les plus remarquables de la MD: «l'enseignement grammatical se greffe[ra] sur nos premiers exercices d'intuition " (Collard 1904 : 30) d'une manière toute nouvelle : l'association des mots aux actions des personnes ${ }^{15}$, à travers la formation de phrases, appelle des points grammaticaux précis, et constitue une acquisition logique et méthodique de la grammaire (Schweitzer 1903b : 17). Ainsi, c'est une réorganisation complète de la grammaire qui laisse de côté l'ordre canonique des règles de phonétique/orthographe, parties du discours, emploi des parties du discours ou syntaxe, déjà ébranlé par les méthodes pratiques du XIX ${ }^{\mathrm{e}}$ siècle. Nous devons ajouter que le germe de cette mise en paralléle se trouve déjà dans le premier manuel Berlitz. En effet, dans MB1-1882a, la présentation des objets permet d'utiliser l'affirmation et la négation (verbe être), la couleur, le féminin des adjectifs, le nombre, le pluriel, la place/position, les prépositions de lieu, le mouvement, les verbes correspondants, etc. Les tableaux synoptiques des manuels Berlitz postérieurs rendent compte précisément de cette démarche, à peu de variations près, tout en complétant dans certaines leçons des lacunes inévitables. La grammaire sera complétée d'autre part dans la partie «Morceaux élémentaires ». Les manuels Berlitz garderont cette logique de base, tout en perfectionnant le modèle (voir MB1-[1915] ${ }^{285} 1924: 8$ ).

Quant à la progression considérée du point de vue psycholinguistique (disons: l'acquisition), nous constatons également une modification de taille, à savoir la distinction de deux phases dans l'enseignement/apprentissage de la langue étrangère : l'intuition directe du sens et l'association des idées. C'est en 1882 que prend forme la méthode Berlitz par la publication simultanée de deux ouvrages: un manuel (MB1-1882a), "pour les commençants», et un Premier livre de lecture qui : «[...] est destiné à remplacer les différents livres de lecture dont nous nous sommes servis jusqu'ici dans nos écoles» (MB- PremierLivre-1882b: préface). À partir de 1887, les morceaux de lecture sont intégrés dans le manuel, dans deux sections différentes:

Documents pour I'histoire du français langue étrangère ou seconde, 64-65 | 2020 
Preparatory Lessons. Object Teaching et Elementary reading and conversations, qui, à partir de l'édition de 1903, seront intitulées respectivement «Partie préparatoire. Leçons de choses » et "Morceaux élémentaires: enseignement par l'association graduelle des idées (Enchaînement) ». Dans le texte qui sert d'introduction au Premier livre de lecture (1882b) nous pouvons lire également: " Quand, après un certain temps, nos élèves ont une pratique suffisante de la langue française, nous leur mettons en mains un livre d'exercices sur les difficultés de cette langue et, plus tard, un cours de composition française et de traduction ». La seconde partie devient en outre plus importante quant à l'espace papier (32, 51,74 pages en 1897, 1903 et 1915). Quant à la composition française, elle sera pratiquée dans le deuxième livre ([MB2- $\left.{ }^{-16} 1901\right]$ et [MBadultes2(1902)1924]). Cette division en deux phases explicite les deux types d'associations qui vont être théorisés par le pédagogue Ferdinand Buisson (1887: Tome 2, 1374-1377) comme composantes de la méthode intuitive :

40 - L'object teaching (les « leçons de choses ») correspond à « l'intuition par les sens. - C'est l'Anschauung proprement dite des pédagogues allemands: on l'a nommée chez nous l'enseignement par l'aspect, ce qui en rétrécit un peu trop le caractère. Les Américains l'ont résumée dans le procédé qu'ils ont assez heureusement nommé object lessons, leçons de choses ";

41 - «l'Intuition dans les facultés intellectuelles», selon l'expression de Buisson, qui correspond, à notre avis, à «l'Enseignement par l'association graduelle des idées (Enchaînement) » de Berlitz.

Buisson souligne également un rapport étroit de la méthode intuitive (en pédagogie) avec le mouvement naturel :

On entend en général par intuition un acte de l'intelligence humaine, le plus naturel, le plus spontané de tous, celui par lequel l'esprit saisit une réalité, constate un phénomène, voit en quelque sorte d'un coup d'œil une chose qui existe en lui ou hors de lui [...] cette vue ne lui coûte ni effort ni réflexion, elle n'entraîne aucune hésitation, elle ne prend pour ainsi dire aucun temps appréciable, tant elle se fait aisément et naturellement (ibid.).

Marcus Reinfried (1990 : 126-156) a établi un antécédent incontestable à la MD dans la démarche d'enseignement mise en œuvre par le mouvement philantropiste de Dessau

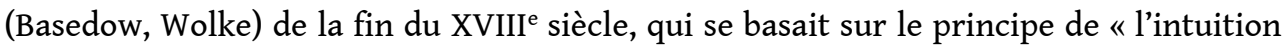
sensible" (versinnlichende Sprachlehrmethode) par la "conception d'une association étroite de l'enseignement des choses à celui des langues étrangères" $(1990: 142)^{16}$. Reinfreid rappelle également que, "dans la première moitié du XIX ${ }^{e}$ siècle, cette méthode naturelle de Dessau avait déjà sombré dans l'oubli »(ibid.), et que les modifications institutionnelles et pédagogiques du système scolaire en Allemagne ainsi que l'influence du courant néo-humaniste dans l'enseignement secondaire, qui allait à l'encontre du sensualisme, va confiner le principe d'intuition à l'enseignement primaire. Il sera repris (avec des modifications) par Johann Heinrich Pestalozzi : «à l'apprentissage [...] de l'esprit d'observation, des exercices de dénomination et de conceptualisation, des descriptions écrites et orales et des exercices grammaticaux en langue allemande » (ibid.: 144). Et c'est à partir de ces expériences des leçons de choses que certains professeurs, dont Karl Griep, eurent l'idée d'utiliser des images dans l'enseignement du français dans les Realschulen (secondaire) au milieu du XIX siècle. C'est donc de ce mouvement, et de Griep en particulier, que Heness avait tiré ses idées de sa méthode naturelle d'enseignement des langues étrangères, qu'il appliqua avec Sauveur dans la French Normal School. 
Pour comprendre la mise en place, par Berlitz, d'une deuxième phase d'enseignement ("L'association graduelle des idées. Enchaînement»), essentielle celle-là pour l'acquisition de la langue étrangère, puisque la première phase n'est que "préparatoire ", nous devons comprendre, comme l'expose Buisson, que la méthode Pestalozzi avait " dégénéré très vite en un nouveau genre de routine et de scolastique " et que les pédagogues postérieurs recommandaient " l'application non de telle ou telle recette plus ou moins mécanique, mais de toutes les méthodes excitatrices de la pensée, pour employer le mot si juste de Diesterweg » (Buisson 1887).

Dans le Mouvement de la Réforme, le concept d'intuition va ainsi s'enrichir, et trois types d'intuitions vont y être distingués : «1ํㅣ'intuition directe de la réalité ou de l'image ; $2^{\circ}$ l'intuition indirecte ou l'évocation de l'image par la mimique; $3^{\circ}$ l'explication de mots nouveaux à l'aide des mots déjà connus » (Schweitzer 1903a : 73). Otto Jespersen parle de son côté de 'direct observation or immediate perception (what the Germans call Anschauung)', 'mediate perception' ou compréhension à l'aide d'images et d'explications (Jespersen $1904: 58$ et 60).

Nous retrouvons ce concept multiple d'intuition chez Berlitz; il met en œuvre dans la partie «Morceaux élémentaires» le principe psychologique de l'association: « l'association des idées, ou explication par le contexte », (MBadultes1 [1903]1905:6) ou « Enseignement par l'association graduelle des idées. Enchaînement ». L'Introduction orale qui accompagne chaque texte - une nouveauté de l'édition de 1915 dont la préface précise: «livres fermés » - et qui permet aux élèves de se familiariser avec les expressions qu'ils vont rencontrer plus tard, la lecture du texte correspondant et les questions qui l'accompagnent, la «conversation» qui s'ensuit et, finalement, les exercices (Appendice 1, édition de 1915) garantissent autant la compréhension que l'expression pour " parler la langue ».

Donc, comme dans la MD, Berlitz complète l'Anschauungmethode par les lectures complémentaires ou Lesebuchmethode (Collard 1904: 36): «Excellente pour la transmission du vocabulaire concret, la méthode intuitive devient insuffisante pour la langue abstraite. Mais là où s'arrête l'intuition commence la lecture » (Simonnot 1901, in Buisson 19117).

Pour résumer notre exposé, l'analyse des manuels signalés dans le corpus ci-dessus nous permet de constater l'existence dès le début d'un nœud didactique central, qui se maintient à grands traits dans les manuels postérieurs analysés (MB1-1882a, MB1PremierLivre-1882b, MB1-[1887]1897/1903], MBadultes1-[1903]1905, MB1-[1915] $\left.{ }^{285} 1924\right)$. Les clés de la méthode Berlitz sont posées en effet dès le premier ouvrage (MB1-1882 : To the Teachers and Students) :

-les cours commencent par une instruction orale ;

-la compréhension de la langue étrangère se fait dans la langue cible elle-même ;

-des exercices de prononciation et de construction sont proposés, qui devront être réalisés de manière attentive, consciente et sérieuse (carefully) ;

-des lectures de textes simples thématiquement ordonnés (la salle de classe, la maison, la ferme, le village, les saisons, etc.);

-des questions de compréhension, qui permettent également à l'élève de s'exprimer en langue cible. 

l'utilisation de l'API, qui d'ailleurs ne fait pas l'unanimité non plus chez les méthodologues directs. On voit ainsi que Berlitz suivait de près ce qui se faisait en Europe, qu'il ne s'est pas enfermé dans ses premiers principes. Le maintien de ces de la pensée avec la langue étrangère sans l'intermédiaire de la langue maternelle. Ces principes prennent corps dans un enseignement par les leçons des choses, par l'association des idées, et un enseignement de la grammaire par exemples et démonstrations.

L'acquisition langagière se produit ainsi grâce à l'immersion langagière, à l'imitation et à la répétition; d'autres idées viennent s'ajouter à partir de 1887 (voir Préface) : l'idée de la systématisation, "nous n'avons fait que systématiser cette façon d'apprendre ", celle d'un séjour à l'étranger, en se démarquant, soit dit en passant, de la méthode "maternelle ", celle aussi du besoin de fixer l'attention des élèves dans les exercices - mais certains exercices écrits sont supprimés, car ils « forçaient les élèves à trop réfléchir »-, enfin des conseils pour « obliger l'élève à imiter la prononciation du professeur $» . .$.

De même, il se produit une évolution dans les exercices : dans MB1-1882a, ils prennent souvent la forme d'un enchâssement d'un mot dans une proposition donnée, les " exercice à trous ${ }^{18}$; à partir de 1887, ils sont remplacés par des « conversations »: bien qu'il s'agisse encore, en réalité, d'exercices, ils prennent toujours la forme d'une interrogation à laquelle l'élève doit répondre avec une phrase complète : nous sommes ainsi près de l'idée de «transformer des connaissances mortes en une pratique vivante, de substituer à un savoir un pouvoir » (Firmery 1902 : 331-332).

Finalement, les outils complémentaires publiés par Berlitz, comme la Grammaire pratique, en quatre volumes, les extraits choisis de littérature, la collection de lectures, les gravures, peuvent être expliqués comme étant des réponses aux jugements négatifs $\mathrm{du}$ Report of the Committee of Twelve évaluant la méthode naturelle et la méthode phonétique (1899:1398-1402). Berlitz, fort de sa longue expérience comme professeurméthodologue en partageait sûrement certains, conscient qu'il était des enjeux de la réforme de l'enseignement-apprentissage des langues vivantes.

\section{Conclusion}

Notre analyse nous a permis de constater une évolution importante dans les manuels Berlitz. Nous avons vu que, à ses débuts, la méthode Berlitz était fortement apparentée à la méthode naturelle. Or, à partir des éditions de 1887, les manuels Berlitz incorporent des principes psycho-pédagogiques de l'acquisition de la langue (l'association des idées, ou explication par le contexte, imitation, répétition, systématisation) qui constituent également les caractéristiques des manuels directs de l'époque : la diminution du vocabulaire à apprendre par cœur (résultat de la distinction entre vocabulaire actif et vocabulaire passif, voir Schweitzer \& Simonnot 1917 : 170-172), le remplacement des exercices par des «conversations », la réduction des leçons préparatoires, l'introduction des lectures complémentaires, l'utilisation des tableaux muraux...

Il reste cependant, que Berlitz ignore les propositions des phonéticiens quant à 
principes et de cette démarche d'enseignement - après leur abandon dans les instructions officielles de l'enseignement secondaire public des divers pays européens et l'implantation de fait d'une "méthode mixte ou éclectique " - doit être signalé comme un fait extrêmement important dans l'histoire de l'enseignement des langues, permettant que ce mouvement rénovateur ne tombe pas dans l'oubli.

\section{BIBLIOGRAPHIE}

\section{Sources primaires ${ }^{19}$}

ALGE, Sines ( $\left.{ }^{5} 1896\right)$. Leçons françaises, première année. Leçons françaises, seconde année. Saint Gall : Librairie Fehr.

ALGE, Sines \& RIPPMANN, Walter (1904). Leçons de français basées sur les tableaux de Hölzel. Saint Gall : Librairie Fehr.

\section{Sources secondaires}

BESSE, Henri (2010). «Irene Finotti. Lambert Sauveur à l'ombre de Maximilian Berlitz. Les débuts de la méthode directe aux Etats-Unis ». Documents pour l'histoire du français langue étrangère ou seconde, 45, 232-239.

BUISSON, Ferdinand ([1887], $2^{\mathrm{e}}$ éd. 1911). Dictionnaire de pédagogie et d'instruction primaire. Paris : Hachette. Tome 2 de la première partie, 1374-1377. Nouvelle édition en 1911. En ligne : [http:// www.inrp.fr/edition-electronique/lodel/dictionnaire-ferdinand-buisson/], (02.02.2018).

COLLARD, François ( $\left.{ }^{3} 1904\right)$. La méthode directe dans l'enseignement des langues vivantes. Bruxelles : Alfred Castaigne.

ESCHER, Erwin (1920). « The 'Invention' of the Natural Method of Language Teaching ». The Modern Language Journal, vol. $4: 6$.

FINOTTI, Irene (2010). Lambert Sauveur à l'ombre de Maximilian Berlitz: Les débuts de la méthode directe aux États-Unis. Bologna : Cooperativa Libraria Universitaria Editrice Bologna. En ligne : [http:// amsacta.unibo.it/2739/1/Finotti_Quaderno_7.pdf].

FIRMERY, Joseph (1902). « La première période de l'enseignement des langues vivantes, d'après les nouveaux programmes ». Revue universitaire, 2, 329-350.

GALISSON Robert \& COSTE, Daniel (1976). Dictionnaire de didactique des langues. Paris : Hachette. GEDDES, James (1930). «Quelques inconvénients des signes et symboles phonétiques ». The French Review, 3, 4, 252-260.

HOWATT, Anthony P. R. \& SMITH, Richard (2014). « The History of Teaching English as a Foreign Language, from a British and European Perspective ». Language \& History, vol. 57 : 1, 75-95.

JESPERSEN, Otto (1904). How to teach a foreign language. London : George Allen \& Unwin Ltd. 
PURIN, Charles M. (1916). « The Direct Teaching of Modern Foreign Languages in American High Schools ». The Modern Language Journal, 1, 2, 43-51.

REINFRIED, Marcus (1990). « Les origines de la méthode directe en Allemagne ». Documents pour l'Histoire du français langue étrangère ou seconde, 6, 126-156. Publié également dans : Herbert Christ \& Daniel Coste (Coord.) 1990. Contributions à l'histoire de l'enseignement du français : actes de la section 3 du Romanistentag d'Aix-la-Chapelle. Tübingen : Gunter Narr Verlag.

ROCHELLE, Ernest (1906). La méthode directe dans l'enseignement des langues vivantes. Bordeaux : G. Delmas.

SCHWEITZER, Charles (1903a). « Communication faite à la Société des Professeurs de Langues vivantes ». Bulletin mensuel - Société des professeurs de langues vivantes de l'enseignement public, 3 (Compte-rendu de la réunion du 2 juillet 1903), 65-89. En ligne : [https://gallica.bnf.fr/ark:/ 12148/bpt6k96858869/f3.item].

SCHWEITZER, Charles (1903b). Méthodologie des langues vivantes. Notes aux conférences faites à la Sorbonne. Paris : A. Colin.

SCHWEITZER, Charles (1904). « Avant-propos ». Enseignement de la langue allemande, Première année, Livre du maître. Paris : A. Colin.

SCHWEITZER, Charles \& SIMONNOT, Émile (1917). Méthodologie des langues vivantes. Paris : A. Colin. SIMONNOT, Émile (1901). « Comment les langues vivantes sont enseignées à l'étranger ». Revue pédagogique, Juillet-Décembre, 18-40.

TITONE, Renzo ([1980] 1985). Glottodidattica. Un profile storico. Bergamo : Minerva Italica.

UNITED STATES. BUREAU OF EDUCATION (1989). Report of the Committee of Twelve of the Modern Language Association of America. Washington, U.S. : Government Printing Office.

WICKERHAUSER, Nathalie (1907). « De la méthode directe dans l'enseignement des langues vivantes ». Die Neuren Sprachen, Band XV, heft 4, 211-232.

\section{NOTES}

1. En ligne : [https://fr.wikipedia.org/wiki/Berlitz], (consulté le 30.03.2019).

2. En ligne : [https://en.wikipedia.org/wiki/Maximilian_Berlitz], (consulté le 30.03.2019).

3. «Le succès le conduit à ouvrir une seconde école à Boston en 1880, avant New York et Washington. Il s'implante dans la foulée à Berlin (1888) et Paris (1889), d'abord rue Auber puis, dans les années 1930, dans un immeuble de bureaux boulevard des Italiens qui prend le nom de palais Berlitz ». En ligne : [https://fr.wikipedia.org/wiki/Berlitz], (consulté le 30.03.2019). Selon un encart du manuel Méthode Berlitz de 1897, il existait 61 écoles Berlitz dans les pays suivants : USA (16), France (5), Angleterre (6), Allemagne (25), Hollande (2), Belgique (2), Autriche-Hongrie (2), Danemark (1), Suisse (1) et Italie (1).

4. Pour rendre plus aisée la consultation des diverses références, nous avons utilisé les abréviations suivantes: MB1-1882a, MB1-PremierLivre-1882b, MB1-[1887]1897/1903, MBadultes1[1903] 1905, MB1-[1915] ${ }^{285} 1924$.

5. En ligne : [https://archive.org/details/mthodeberlitzp0oberluoft/page/n1/ mode/2up].

6. En ligne : [https://archive.org/details/premierlivrepourooberluoft/page/n1/ mode/2up]. 
7. «Méthode directe » au sens de « ensemble de procédés d'enseignement des langues étrangères qui s'est constituée, au début du siècle, en relation avec l'évolution des théories éducatives » (Galisson \& Coste 1976 : 154).

8. Comme le soulignent Howatt \& Smith (2014: 83), cette reconnaissance portée à Heness disparaît dans les éditions postérieures du manuel.

9. Ainsi que Kroeh et van Daell (cités par Purin $1916: 46$ ).

10. Le Report of the Committee of Twelve [...], publié par le Gouvernement des USA (United States Bureau of Education) en 1899, et élaboré à l'initiative de la Modern Language Association of America, trace un profil détaillé de l'enseignement des langues étrangères aux USA dans la seconde moitié du XIX ${ }^{\mathrm{e}}$ siècle, que nous n'avons point l'espace ici de résumer.

11. Pour reprendre une opinion de l'époque (celle de Charles Purin), la méthode naturelle se différenciait de la MD par ces trois aspects :

1. It lacks the phonetic basis.

2. In its first stages it makes no use of reading or writing, but deals with conversation pure and simple.

3. It postpones to a very late period statements of connected grammatical principles (Purin 1916 : 46).

12. Cela constitue un fait exceptionnel, puisque tous les autres manuels seront signés uniquement par M.D. Berlitz.

13. Remarquons que dans la MD c'est une progression similaire qui sera conseillée (voir Schweitzer 1903b : 15).

14. Ce n'est que dans l'édition de 1915 que le Premier livre de la méthode Berlitz mentionne l'utilisation de gravures.

15. Schweizer remarque justement que la méthode directe «associe les mots aux actions des êtres » $(1904: \mathrm{V})$.

16. Cette démarche inspirée de l'Orbis pictus de Comenius, s'en différencie par la pratique du monolinguisme, donc, de l'accès au sens direct (Reinfried 1990 : 128).

17. En ligne: [http://www.inrp.fr/edition-electronique/lodel/dictionnaire-ferdinand-buisson/ document.php?id=3019].

18. Par exemple : «Votre paletot est brun ? Oui, M...., - paletot est - - brun ». L'apprenant doit remplacer les ... par une partie d'un mot, un trait d'union - par un mot, deux traits d'union - par deux mots, etc.

19. Pour les manuels de Berlitz, voir le corpus présenté en début d'article.

\section{RÉSUMÉS}

Cet article décrit la méthode Berlitz, à travers l'analyse des manuels pour l'enseignement du français (Premier livre) publiés entre 1882 (première édition) et 1915. L'analyse se structure en trois parties : l'établissement des publications constituant les matériels langagiers d'enseignement (pour la langue française), et donc, du corpus ; l'analyse des manuels, qui met en lumière l'évolution de la méthode ; la conclusion. L'une des conclusions de cette étude montre que la méthode Berlitz, issue à l'origine de la méthode naturelle telle qu'elle était pratiquée aux États-Unis (G. Heness, L. Sauveur), se rapproche par la suite de la « méthode directe » : ainsi, la démarche initiale, basée sur l'intuition directe (« leçons de choses », Anschauung), est complétée 
par la démarche d'« enseignement par l'association graduelle des idées (Enchaînement) ", qui correspond au principe direct de la mediate perception (compréhension ou interprétation par le contexte, association).

This article describes the Berlitz method, through the analysis of the textbooks for the teaching of French (First book) published between 1882 (first edition) and 1915. The analysis is structured in three parts: the establishment of the publications constituting the language teaching materials for the French language, and therefore, the corpus; textbook analysis, which highlights the evolution of the method; conclusion. One of the conclusions of this study shows that the Berlitz method, originally derived from the natural method as it was practiced in the United States (G. Heness, L. Sauveur), subsequently approximates the "Direct method": thus, the initial approach, based on direct intuition ("object teaching", Anschauung), is supplemented by the approach of "Teaching by the gradual association of ideas", which corresponds to the direct principle of "mediate perception" (understanding or interpretation by the context, association).

\section{INDEX}

Keywords : natural method, direct method, USA, Heness, Sauveur, intuition, object teaching, Report of the Committee of Twelve

Mots-clés : méthode naturelle, méthode directe, USA, Heness, Sauveur, intuition, leçons de choses, Report of the Committee of Twelve

\section{AUTEUR}

\section{JAVIER SUSO LÓPEZ}

Université de Grenade - jsuso@ugr.es 